\title{
COMPORTAMIENTO AGRONÓMICO DE LÍNEAS PARA LA PRODUCCIÓN DE SEMILLA DE SORGO ${ }^{1}$
}

\author{
Antonio Flores-Naveda ${ }^{2}$,Ciro G.S. Valdés-Lozano², Francisco Zavala-García ${ }^{2}$,Emilio Olivares-Sáenz², \\ Adriana Gutiérrez-Díez ${ }^{2}$, Mario Ernesto Vázquez-Badillo
}

\section{RESUMEN}

Comportamiento agronómico de líneas para la producción de semilla de sorgo. El objetivo de esta investigación fue evaluar el comportamiento agronómico de líneas isogénicas A, B y líneas R restauradoras de la androfertilidad de sorgo para grano. El ensayo se realizó durante el ciclo primavera-verano 2009-2010 en Marín Nuevo León, México, bajo riego por goteo. Se evaluaron días a floración, rendimiento de semilla en gramos por planta, grados Brix en el jugo del tallo y peso de 1000 semillas. Se realizó el análisis estadístico con la prueba de comparación de medias con muestras independientes en las líneas isogénicas A y B. En las líneas R, se realizó el análisis de varianza y la prueba DMS $(\mathrm{P}<0,05 \%)$. Algunas de las líneas A y $\mathrm{B}$ no fueron isogénicas. Se encontraron líneas A y $\mathrm{R}$ que en cruza pueden formar híbridos para la producción comercial de grano de sorgo. Se identificaron líneas B y R que pueden utilizarse como variedades y se definieron los días de siembra para sincronizar la floración de las líneas progenitoras A y R para la producción de semilla híbrida de sorgo.

Palabras clave: Sorghum bicolor, grano de sorgo, líneas isogénicas, variedades, híbridos.

\begin{abstract}
Agronomic performance of sorghum seed production lines. The objective of the study was to evaluate the agronomic performance of $\mathrm{A}, \mathrm{B}$ isogenic lines and $\mathrm{R}$ grain sorghum lines which restore androfertility of sorghum for grain production. This work was performed during the spring-summer 2010 in Marin Nuevo Leon under drip irrigation. Days to flowering, seed yield per plant, brix degrees and weight of 1000 seeds were measured. The statistical analysis with the benchmark test of averages with independent samples was realized in A, B sorghum lines to days to flowering, seed yield per plant, brix degrees and weight of 1000 seeds. In $\mathrm{R}$ lines the analysis of variance and DMS $(\mathrm{P}<0.05 \%)$ test was performed. A an B lines were not completely isogenic; there are $\mathrm{A}$ and $\mathrm{R}$ lines that may be crossed to produce commercial sorghum grain seed. $\mathrm{B}$ and $\mathrm{R}$ lines might be used as varieties and $\mathrm{A}, \mathrm{R}$ parental lines planting splits were defined to produce hybrid sorghum seed.
\end{abstract}

Key words: Sorghum bicolor, grain sorghum, isogenic lines, cultivars, hybrids.

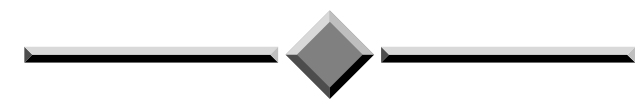

1 Recibido: 9 de febrero, 2012. Aceptado: 1 de abril, 2013. Este trabajo forma parte de la Tesis de doctorado del primer autor, realizada en la Facultad de Agronomía de la Universidad Autónoma de Nuevo León, México.

2 Facultad de Agronomía. Universidad Autónoma de Nuevo León. Campus Ciencias Agropecuarias Francisco Villa s/n. C.P. 66050. Col. Ex-hacienda. El Canadá. Escobedo, Nuevo León. México. Tel: 018113404399. naveda26@ hotmail.com, cigsvalloz@ hotmail.com, francisco. zavalagr@uanl.edu.mx, emolivares@gmail.com,mcgudiez@hotmail.com

3 Departamento de Fitomejoramiento. Universidad Autónoma Agraria Antonio Narro. Buenavista, Saltillo, Coahuila, México. C.P. 25315. mario59ernesto@hotmail.com

(i) $\odot$ (c) 2013 Agronomía Mesoamericana es desarrollada en la Universidad de Costa Rica y se encuentra licenciada con Creative 


\section{INTRODUCCIÓN}

El cultivo de sorgo (Sorghum bicolor (L.) Moench) es el quinto cereal más importante del mundo después del trigo, maíz, arroz y cebada (FAO 2011). A nivel mundial, el sorgo es cultivado en una superficie de 42 millones de hectáreas con una producción anual de 58,5 millones de toneladas y es utilizado en la dieta alimenticia de 500 millones de personas en más de 30 países (ICRISAT 2009). En Asia y África es utilizado como cultivo alimenticio (Gous 1989, Anglani 1998, Dykes et al. 2005, Dicko et al. 2006). Actualmente en el Salvador se emplea harina de sorgo para la industria de la panificación (CENTA 2012).

El noreste de México es la región con mayor producción de sorgo del país, en donde se siembran anualmente más de un millón de hectáreas (Williams et al. 2006). La superficie sembrada en esta región, durante el año agrícola 2010 fue de 932054 hectáreas en la modalidad de riego más temporal con un rendimiento promedio de 2,9 t/ha (SIAP-SAGARPA 2010).

Para la producción de semilla híbrida de sorgo se utiliza un sistema de tres líneas denominadas A, B y R; donde la A es androestéril y la B es androfértil; de tal forma que la progenie de la cruza A x B (línea A materna y B paterna), es estéril (Rao 2002); la esterilidad masculina es el resultado de factores en el citoplasma, el cual es heredado en forma maternal y la línea B es isogénica que tiene un citoplasma normal, pero carece de genes restauradores, por lo cual, la cruza A x B permite mantener o incrementar la línea $\mathrm{A}$, conservándose androestéril.

Para el desarrollo de híbridos de sorgo es necesaria la evaluación del comportamiento per se de las líneas, así como el de su actitud combinatoria general (ACG) y específica (ACE) (Poehlman 2005).

La línea $\mathrm{R}$ es capaz de restaurar la fertilidad en la cruza A x R, en donde estas líneas se seleccionan, por su alta capacidad para producir una progenie híbrida en donde se presente un aceptable vigor para rendimiento de grano. A su vez, la producción de semilla híbrida de sorgo involucra a una línea $\mathrm{R}$ restauradora y una línea $\mathrm{B}$ mantenedora como fuentes de polen, las $\mathrm{R}$ producen más polen viable que las B (Cisneros-López et al. 2012).

Las líneas $\mathrm{R}$ restauradoras deben presentar una gran producción y un mayor tiempo de producción del polen, para tener una polinización eficiente en el campo de producción de semilla y distribuir los arreglos de siembra $3: 1 ; 4: 2 ; 12: 4$ hembra-macho. Murty (1999) recomienda, para una adecuada producción de semilla híbrida de sorgo a escala comercial, la siembra de dos surcos machos alternados con cuatro surcos hembra, cuando la línea macho presenta panícula pequeña y floración corta, o dos surcos macho con seis surcos hembras, cuando la línea macho presenta panícula grande y floración prolongada.

La planta de sorgo es predominantemente autógama por lo tanto, presenta un promedio de cruzamiento del $4 \%$ aunque pueden ocurrir niveles significativos de cruza natural de un $10 \%$ de cruzamiento para panículas con forma compacta (House 1985), por lo tanto, el cultivo está expuesto a perder la pureza original, debido a posible contaminación por otras fuentes de polen, por lo cual es necesario producir la semilla en lotes aislados, para mantener su pureza genética (Poehlman 2005) y garantizar la calidad de la semilla (García y Guiragossian 1985).

La integridad genética de una línea de sorgo se mantiene mediante la autofecundación, cuando se regeneran gran cantidad de accesiones simultáneamente y no es posible aislarlas, es necesario cubrir las panículas de sorgo con bolsas de papel, previo a la antesis para mantener su pureza en la producción de semilla básica. La semilla original conserva los caracteres que le dan superioridad a una variedad y constituye la fuente inicial para la producción de semillas en las categorías básica, registrada y certificada (SNICS 2007).

La semilla es la portadora del potencial genético que determina la productividad y constituye el insumo más importante para alcanzar altos rendimientos en cualquier cultivo. Esta es el resultado de la fertilización, desarrollo y maduración del óvulo y está formada por testa, endospermo y embrión. Una producción de semilla aceptable está ligada a una buena calidad fisiológica, la cual determina la capacidad de germinación y el establecimiento de las plántulas durante sus primeras etapas vegetativas.

La semilla de alta calidad es uno de los insumos más importantes para asegurar un buen establecimiento de plántulas en campo y con ello la densidad de población óptima para alcanzar un alto rendimiento, aunado a esto la emergencia y establecimiento están influenciados por los factores ambientales de temperatura, humedad del suelo (Helms et al. 1997) y por los aspectos de calidad física, fisiológica, genética y sanitaria de la semilla (Finch-Savage 1995). 
En México la disponibilidad de semilla de sorgo para siembra depende prácticamente de la híbrida importada y su costo en el mercado es cada vez más alto. Por lo tanto, se utiliza la híbrida que es producida principalmente en los EEUU y comercializada en México, lo que indica que nuestro país es dependiente de este insumo. El costo es de siete dólares por kilogramo.

Anualmente se siembran alrededor de dos millones de hectáreas de sorgo para grano en el país (SIAP 2011), prácticamente toda esta superficie se cultiva con híbridos y las siembras con variedades de polinización libre, no son significativas; no obstante, la principal ventaja de una variedad de sorgo es que la multiplicación de semilla la podrían realizar agricultores capacitados en producción de semilla de calidad, y por lo tanto, se reduciría el costo en la producción de esta, haciendo este insumo más accesible a los productores.

En el noreste de México predominan las siembras con semillas híbridas de sorgo, representando este insumo un alto costo en el mercado de semillas mejoradas, y a la vez estos híbridos son desarrollados para ambientes favorables al cultivo, por lo tanto, una alternativa es utilizar semilla de variedades tipo línea pura (Valdés et al. 2004) ya que es más económica y fácil de producir, por lo tanto puede ser utilizada por los agricultores en sus próximos ciclos agrícolas, además las líneas pueden competir con los híbridos en ambientes no favorables, si son seleccionadas bajo estos ambientes, pudiendo incluso superar en rendimiento de grano a los híbridos comerciales (Flores y Valdés 2009). Por lo tanto, el objetivo del presente estudio fue evaluar el comportamiento agronómico y estabilidad de líneas A, B y R de sorgo para grano.

\section{MATERIALES Y MÉTODOS}

El presente estudio se realizó durante el ciclo primavera-verano 2009-2010, en ocho bancales bajo riego por goteo en Marín, Nuevo León, México. Su ubicación geográfica es $25^{\circ} 52^{\prime} 13,5^{\prime \prime}$ latitud norte y $100^{\circ} 02^{\prime} 22,56^{\prime \prime}$ longitud oeste, a una altura de 355 msnm. El clima corresponde a un BS1 (h) w (e), descrito como clima seco estepario cálido con lluvias en verano, una precipitación promedio anual de $595 \mathrm{~mm}$ y una temperatura promedio anual de $22^{\circ} \mathrm{C}$. El tipo de suelo es calcáreo, arcilloso con un pH de 7,5 con bajos contenidos de materia orgánica (García 2004).
Se utilizó como germoplasma un grupo de líneas de sorgo desarrolladas en el Programa de Mejoramiento de Sorgo de la Facultad de Agronomía de la Universidad Autónoma de Nuevo León (FAUANL) en Marín, Nuevo León. Las líneas R: 10475, 10351, 90538, 90520, LES40 R, LES88, LES124, LES150, LES151, LES154, LES162 y LES163. Líneas isogénicas A, B de sorgo: 398 A,B; 1823 A,B; 1829 A,B; 1831 $\mathrm{A}, \mathrm{B} ; 1832 \mathrm{~A}, \mathrm{~B}$ en tres fechas de siembra (FS) FS1= 4 de agosto, FS2 $=7$ de agosto y FS3= 11 de agosto. Las parcelas experimentales se establecieron en cuatro surcos de 2 x 0,25 metros (m), con espacios de $0,6 \mathrm{~m}$ por cada parcela para las líneas $\mathrm{R}$ y para los pares A, B se establecieron dos parcelas de 2 x $0,25 \mathrm{~m}$, con los dos surcos centrales de línea A y dos laterales de la línea B.

En las etapas iniciales del cultivo de sorgo como parte del manejo agronómico se realizó un raleo de plántulas, con la finalidad de establecer una densidad de 10-12 plantas por metro lineal, durante el desarrollo del cultivo se realizó el control de malezas mediante escardas. Para el control de plagas se aplicó cipermetrina 200 concentrado emulsionable. La fertilización se realizó aplicando fertilizante granulado en banda de 18-46-0 y aplicaciones foliares con la fórmula 2030-10. Previo a la floración se identificaron las plantas fuera de tipo y se procedió a realizar su desmezcle. Durante la etapa de floración se realizaron aplicaciones preventivas con un fungicida agrícola a base de propiconazol, para evitar incidencia e infestación de las inflorescencias por Ergot del sorgo (Claviceps africana).

Para realizar el incremento de las líneas A, B se procedió a la polinización manual de cada línea A con polen de su línea isogénica B mantenedora y la autofecundación de esta última, procurando que las plantas A y $B$ fueran semejantes entre ellas. Para lo anterior, en el incremento de las líneas A, se procedió a cubrir la panícula con una bolsa de glacine al inicio de la apertura de las flores y de la misma manera se procedió con las líneas B poco antes del inicio de la antesis. Cuando las panículas de las líneas A, habían alcanzado el 90 o 100\% de estigmas receptivos, se procedió a la polinización con polen de la línea isogénica $\mathrm{B}$ correspondiente, manteniendo posteriormente las panículas de la línea B cubiertas para obtener semilla de autofecundación. En las líneas R, se cubrieron las panículas al inicio de antesis, para evitar contaminación de alguna fuente de polen extraño y de esta manera obtener semilla de autofecundación. 
La fecha de inicio de las cruzas para el incremento de semilla y mantenimiento de las líneas isogénicas de sorgo se realizaron al momento del inicio de antesis, el día 6 de octubre del 2009 en el Bancal 8 parcela 1, en las líneas isogénicas 1832 A y B. En líneas R, solo se cubrieron las panículas al inicio de antesis para evitar contaminación por alguna fuente de polen extraño y asegurar la pureza genética de las líneas con semilla de autofecundación. La cosecha de las panículas de sorgo se realizó en forma manual el día 14 de diciembre del 2009.

\section{Variables evaluadas}

Días a floración. Se evaluó en el momento en que las plantas de sorgo presentaban el 50\% de las panículas en antésis media.

Grados Brix. Se evaluaron dos muestras por planta en cada una de las parcelas del área de Bancales, en donde se tomó la muestra del jugo del tallo y se colocó en el refractómetro portátil, para observar la lectura correspondiente de la concentración de azúcares en grados Brix.

Rendimiento de semilla en gramos por planta. Se realizó el trillado de las panojas de sorgo para obtener el rendimiento de semilla en las líneas A y B, $\mathrm{R}$ y se registró el peso total dividido entre el número de panículas por parcela para obtener el rendimiento promedio en gramos de semilla por planta.

Peso de 1000 semillas. Se tomó una muestra de grano para determinar el peso de mil semillas en tres repeticiones y después se procedió a registrar su peso en gramos.

El análisis estadístico se realizó por la prueba de comparación de medias con muestras independientes en las líneas isogénicas A, B de sorgo para las variables días a floración, grados Brix, gramos de semilla por planta y peso de 1000 semillas.

En las líneas $\mathrm{R}$ de sorgo se realizó el análisis de varianza, bajo un diseño completamente al azar con diferente número de repeticiones, equivalentes a diferentes números de plantas evaluadas y para las variables donde se detectó diferencia significativa, se utilizó la prueba de comparación de medias por diferencia mínima significativa (DMS) $(\mathrm{P} \leq 0,05 \%)$ para días a floración, grados Brix, gramos de semilla por planta y peso de 1000 semillas con el paquete de cómputo de diseños experimentales FAUANL, versión 2.5 (Olivares 1994).

\section{RESULTADOS Y DISCUSIÓN}

\section{Líneas isogénicas A, B de sorgo}

En el Cuadro 1, se observa que cuatro líneas isogénicas $\mathrm{A}, \mathrm{B}$ presentan sincronización floral, por lo tanto, es un factor fundamental en la producción de semilla de sorgo (Asfaw y Tesfaye 2008), excepto la línea 1832 A, B, en donde la línea B presenta tres días más a floración que su línea isogénica $\mathrm{A}$, por tanto, debe corregirse mediante selección en próximos ciclos agrícolas, para definir los días de siembra de estas líneas progenitoras con la finalidad de sincronizar la floración, de acuerdo con la información obtenida en esta investigación. En general, las líneas evaluadas, se encuentran dentro del rango de 55 a 70 días a floración en sorgo, tal como lo reporta (House 1985). Durante la floración, las glumas abren y las tres anteras caen libres, mientras los dos estigmas sobresalen cada uno en un estilo rígido. El periodo de tiempo desde que la gluma abre hasta terminar de cerrarse, es alrededor de una o dos horas, esto varía de acuerdo al genotipo (House 1985).

El polen de sorgo permanece viable solo por unas horas, por el contrario, los estigmas permanecen viables por diez días, aunque esto depende del ambiente; sin embargo, la receptividad del estigma se incrementa al quinto día, después de la floración (Puttarudrappa y Goud 1970, Patil y Goud 1980) y disminuye gradualmente. Por su parte, Schertz y Dalton (1980) reportan que bajo condiciones normales la fertilización de un estigma receptivo se realiza en dos horas, la diferenciación del órgano ocurre en los siguientes doce días y el embrión y/o óvulo fecundado y maduro, continúa creciendo hasta alcanzar la madurez fisiológica de la semilla.

En el Cuadro 1 se observa que las líneas isogénicas A, B presentan valores promedios en un rango de 9,6 a 17,4 para contenido de grados Brix en el jugo del tallo, siendo valores aceptables para plantas de sorgo con alta cantidad de jugo y una alta concentración de azúcares, de acuerdo a lo mencionado por Pfeiffer $e t$ al. (2010) que reportan contenidos de 12 a 22 grados Brix en concentración de azúcares en sorgo. Los azúcares contenidos en el jugo del tallo, se acumulan durante el desarrollo de la inflorescencia y en la madurez del grano, los principales son la sacarosa como disacárido y la glucosa y fructosa como monosacáridos (Muminov 1997, Hoffman-Thoma et al. 1996). 
Cuadro 1. Prueba de comparación de medias con muestras independientes en líneas isogénicas A, B de sorgo para grano. En Marín, Nuevo León, México. Ciclo primavera-verano 2009.

\begin{tabular}{|c|c|c|c|c|c|c|c|c|}
\hline \multirow[t]{2}{*}{ Línea } & \multicolumn{2}{|c|}{ Días a floración } & \multicolumn{2}{|c|}{ Grados Brix } & \multicolumn{2}{|c|}{$\begin{array}{c}\text { Gramos de semilla por } \\
\text { planta }\end{array}$} & \multicolumn{2}{|c|}{$\begin{array}{c}\text { Peso de } \\
1000 \text { semillas }\end{array}$} \\
\hline & Media & Comparación & Media & Comparación & Media & Comparación & Media & Comparación \\
\hline $398 \mathrm{~A}$ & 60,6 & $A=B$ & 17,3 & $\mathrm{~A}>\mathrm{B}$ & 22,9 & $A=B$ & 30,3 & $\mathrm{~A}>\mathrm{B}$ \\
\hline $398 \mathrm{~B}$ & 60,0 & & 12,6 & & 23,6 & & 27,7 & \\
\hline $1823 \mathrm{~A}$ & 64,2 & $\mathrm{~A}=\mathrm{B}$ & 15,2 & $A=B$ & 33,2 & $\mathrm{~A}<\mathrm{B}$ & 26,3 & $A=B$ \\
\hline 1823 B & 62,2 & & 13,8 & & 41,4 & & 27,9 & \\
\hline 1829 A & 63,7 & $\mathrm{~A}=\mathrm{B}$ & 16,2 & $A=B$ & 32,8 & $\mathrm{~A}<\mathrm{B}$ & 25,9 & $\mathrm{~A}<\mathrm{B}$ \\
\hline 1829 B & 62,0 & & 17,4 & & 44,9 & & 28,3 & \\
\hline $1831 \mathrm{~A}$ & 68,5 & $A=B$ & 16,2 & $A=B$ & 22,1 & $\mathrm{~A}<\mathrm{B}$ & 33,9 & $\mathrm{~A}>\mathrm{B}$ \\
\hline $1831 \mathrm{~B}$ & 68,1 & & 14,0 & & 71,4 & & 26,8 & \\
\hline $1832 \mathrm{~A}$ & 63,1 & $\mathrm{~A}>\mathrm{B}$ & 13,8 & $\mathrm{~A}>\mathrm{B}$ & 37,7 & $A=B$ & 32,1 & $\mathrm{~A}>\mathrm{B}$ \\
\hline 1832 B & 60,8 & & 9,6 & & 40,3 & & 27,5 & \\
\hline
\end{tabular}

Línea isogénica $\mathrm{A}, \mathrm{B}$ = no hay diferencia de comparación en el carácter evaluado; $\mathrm{A}>\mathrm{B}$, la línea A presenta una tendencia mayor de comparación, respecto a su línea isogénica B; A $<\mathrm{B}$ la línea A presenta una tendencia menor de comparación, respecto a su línea isogénica $B$.

La determinación de grados Brix, muestra el contenido de sólidos solubles en el jugo de la planta de sorgo. El porcentaje de azúcares está estrechamente relacionado con las condiciones climáticas las cuales directa e indirectamente afectan los procesos fisiológicos en la planta, los cuales determinan el contenido de azúcares.

Los valores reportados en este estudio para contenido de grados Brix, se evaluaron durante la etapa de madurez del grano como lo recomienda Ferrari (1986). Esto explica la alta concentración de azúcares en las líneas A, B de sorgo y por tanto coincide con Lingle (1987) el cual reporta, que la madurez fisiológica del grano es la etapa óptima para la cosecha.

Para la variable rendimiento de semilla en gramos por planta (Cuadro 1), tres líneas isogénicas A, $\mathrm{B}$ presentaron un rendimiento promedio similar para esta variable. En líneas A de sorgo se debe presentar un alto grado de esterilidad masculina, así como un rango máximo de estigmas receptivos al polen para garantizar una buena producción de semilla (Williams et al. 2006). La línea 398 A de sorgo, puede explotar heterosis para la producción de semilla híbrida, la cual puede manifestarse mediante floración más precoz, mayor número de hojas, peso, tamaño de semilla y producción de grano (Tüsüz 1997), mayor velocidad de emergencia, vigor y peso seco de plántula (Yu y Tuinstra 2001, Cisneros-López et al. 2007). Un híbrido de sorgo explota la heterosis de la cruza de una línea $\mathrm{A}$ androestéril con una línea $\mathrm{R}$ fértil restauradora de la fertilidad masculina.

Las líneas B, por ser plantas fértiles tienden a producir una mayor cantidad de polen y por tanto, una mayor cantidad de semilla de autofecundación, aunque el peso y tamaño de esta depende de la habilidad de la planta para acumular materia seca durante la etapa de llenado del grano (Cuadro 1).

Para la variable peso de 1000 semillas, las líneas 398,1831 y 1832 A presentaron valores promedio de 30,3, 33,9 y 32,1 gramos, respectivamente (Cuadro 1). Estas líneas se encuentran en el promedio para el cultivo de sorgo de 20 a 35 gramos (ISTA 2003). Las diferencias en peso mayor para las líneas A, se atribuye a que la semilla de las líneas androestériles, se obtuvo mediante cruzamientos manuales (A x B) donde normalmente no se obtiene $100 \%$ de éxito en la polinización, por lo que las semillas desarrolladas en la 
panícula son pocas, pero con mayor peso y tamaño que las producidas mediante polinización libre.

\section{Líneas $\mathbf{R}$ de sorgo}

Para días a floración en líneas $\mathrm{R}$, el análisis de varianza detectó diferencia significativa entre tratamientos, por tanto, se realizó la comparación de medias (Cuadro 2), en donde se observa que la variedad más tardía fue la línea 10475 con 68 días y la más precoz fue LES124 R con 58 días. Las líneas R se encuentran en un rango de 58 a 68 días a floración, tal como lo reporta House (1985), donde el sorgo inicia floración de 55 a 70 días en climas cálidos, aunque depende del genotipo. En general, la iniciación floral en sorgo cultivado comienza a los 30 y 40 días después de la germinación de la semilla, la flor inicia su desarrollo en una inflorescencia, y tarda alrededor de 6 a 10 días, antes de la floración, en donde se forma una bota o envoltura que sobresale en la vaina de la hoja bandera.

Una línea $\mathrm{R}$ restauradora de polen como polinizador, debe presentar un gen dominante restaurador de la fertilidad y genes modificadores necesarios para complementar la restauración de la fertilidad en el híbrido F1. A su vez, debe producir abundante polen fértil y cruzarse con la línea A para generar un híbrido de cruza simple de alto rendimiento con calidad de grano. La línea $\mathrm{R}$ es androfértil, posee genes restauradores de la fertilidad y se combina con una línea A para producir semilla híbrida de cruza simple. Por otro lado, una buena sincronización de la floración entre progenitores masculino y femenino es de suma importancia para asegurar una eficiente polinización y con ello disminuir el porcentaje de infección del ergot del sorgo en lotes de producción de semilla.

En el Cuadro 2 se observa que las líneas R presentaron un rango de 10,2 a 18,8 para contenido de grados Brix del jugo del tallo, siendo estos valores aceptables para plantas de sorgo dulce, de acuerdo con lo mencionado por Pfeiffer et al. (2010).

Para la variable rendimiento de semilla por planta en líneas $\mathrm{R}$, el análisis de varianza detectó diferencia significativa entre tratamientos. Por lo tanto, se realizó la comparación de medias, en donde se observa que la línea 90538 presentó el mayor rendimiento promedio con 62,7 gramos (Cuadro 2).

El análisis de varianza para la variable peso de 1000 semillas en líneas $\mathrm{R}$ de sorgo, detectó diferencia significativa entre tratamientos. La línea 90538 presentó el mayor peso de 1000 semillas con un promedio de

Cuadro 2. Comparación de medias en líneas R de sorgo para grano en Marín, Nuevo León, México. Ciclo primavera-verano 2009.

\begin{tabular}{|c|c|c|c|c|c|c|c|c|c|}
\hline \multirow{2}{*}{$\begin{array}{l}\text { Línea R } \\
10475\end{array}$} & $\begin{array}{c}\text { Días a } \\
\text { floración }\end{array}$ & \multirow{2}{*}{$\begin{array}{c}\text { Línea R } \\
90520\end{array}$} & \multirow{2}{*}{$\begin{array}{l}\text { Grados Brix } \\
18,8 \mathrm{a}\end{array}$} & \multirow{2}{*}{$\begin{array}{c}\text { Línea R } \\
90538\end{array}$} & \multicolumn{2}{|c|}{$\begin{array}{l}\text { Gramos de semi- } \\
\text { lla por planta }\end{array}$} & \multirow{2}{*}{$\begin{array}{c}\text { Línea R } \\
90538\end{array}$} & \multicolumn{2}{|c|}{$\begin{array}{c}\text { Peso de } 1000 \\
\text { semillas }\end{array}$} \\
\hline & $68,3 \mathrm{a}$ & & & & 62,7 & $\mathrm{a}$ & & 36,7 & $\mathrm{a}$ \\
\hline LES151 & $67,6 \mathrm{ab}$ & LES154 & $18,2 \mathrm{a}$ & 10475 & 61,1 & $\mathrm{a}$ & 10351 & 34,0 & $\mathrm{~b}$ \\
\hline LES154 & $67,6 \mathrm{ab}$ & LES40 & $17,7 \mathrm{ab}$ & 10351 & 51,1 & $\mathrm{ab}$ & LES163 & 33,2 & $\mathrm{bc}$ \\
\hline 90538 & $64,3 \mathrm{abc}$ & LES163 & $17,6 \mathrm{ab}$ & LES151 & 42,3 & $\mathrm{bc}$ & LES162 & 32,9 & $\mathrm{bc}$ \\
\hline LES40 & $63,6 \mathrm{abc}$ & LES124 & $17,2 \mathrm{ab}$ & 90520 & 41,1 & $\mathrm{bc}$ & 90520 & 32,5 & $\mathrm{c}$ \\
\hline 90520 & $63,0 \quad$ bcd & LES162 & $17,0 \mathrm{ab}$ & LES162 & 40,6 & $\mathrm{bc}$ & LES124 & 32,1 & $\mathrm{c}$ \\
\hline LES163 & $63,0 \quad$ bcd & LES151 & $14,6 \mathrm{bc}$ & LES163 & 37,0 & $\mathrm{bc}$ & LES88 & 28,5 & d \\
\hline LES 162 & 62,0 & 10351 & $14,4 \mathrm{bc}$ & LES154 & 33,6 & c & LES150 & 27,8 & d \\
\hline LES150 & 62,0 & LES88 & $13,3 \mathrm{bcd}$ & LES40 & 32,2 & $\mathrm{c}$ & LES154 & 27,8 & d \\
\hline 10351 & 61,3 & 90538 & $12,2 \mathrm{bcd}$ & LES150 & 29,5 & $\mathrm{c}$ & 10475 & 27,7 & d \\
\hline LES88 & 58,3 & LES150 & $11,5 \mathrm{~cd}$ & LES88 & 27,4 & $\mathrm{c}$ & LES40 & 24,3 & $\mathrm{e}$ \\
\hline LES124 & 58,0 & 10475 & 10,2 & LES124 & 13,7 & $\mathrm{~d}$ & LES151 & 20,8 & f \\
\hline
\end{tabular}

Tratamientos con la misma letra son estadísticamente iguales, tratamientos con letra distinta, indican diferencias significativas entre ellos. 
36,7 gramos y la línea LES151 R presentó un peso mínimo de 20,8 g (Cuadro 2) en un rango promedio para el cultivo de sorgo de 20 a 35 gramos (ISTA 2003).

En el presente trabajo, del grupo de líneas evaluadas se realizó un análisis conjunto del rendimiento de semilla por planta en gramos de las líneas B y R de sorgo, encontrándose diferencias significativas entre los tratamientos evaluados, por lo tanto, se realizó la comparación de medias. Se observó que la línea 1831B con $71,3 \mathrm{~g}$ de semilla por planta y las líneas $90538 \mathrm{R}$, 10475R presentaron el mayor rendimiento, comparado con la línea 398B que presentó el menor rendimiento con 23,6 g, estas diferencias en peso están asociadas a que las líneas 1831B, 90538R, 10475R son más tardías que la 398B. Estos rendimientos por planta indican un potencial aceptable para la utilización de estas líneas como variedades.

Mediante el conocimiento de los días a floración de las líneas A y R se definieron los días de siembra de las líneas progenitoras con la finalidad de sincronizar la floración para producir semilla híbrida de sorgo. Se observó que tres combinaciones de las líneas progenitoras A x R, podrían sembrarse el mismo día y trece cruzas de la línea $R$, se tienen que sembrar un día antes de la línea A. Para el grupo restante de líneas el día de siembra se incrementa y solo se presentan dos cruzas con once días a la siembra, lo que dificulta la sincronización floral de los progenitores A x R para la producción de semilla híbrida. Se identificaron días de siembra con diferencias mínimas en la sincronización de los días a floración entre las líneas progenitoras que permiten una fácil producción de semilla híbrida de sorgo para grano a escala comercial.

\section{LITERATURA CITADA}

Anglani, C. 1998. Sorghum for human food. A review. Plant Foods Hum. Nutr. 52(1):85-95.

Asfaw, A; Tesfaye, T. 2008. Seed production potential of ICRISAT bred parental lines of two sorghum hybrids in the central Rift-Valley of Ethiopia. Journal of SAT Agricultural Research. 6 p.

CENTA (Centro Nacional de Tecnología Agropecuaria y Forestal). 2012. El sorgo para elaboración de alimentos. División de Comunicaciones CENTA. Cuidad Arce, La Libertad. El Salvador (en línea). Consultado marzo 2012. Disponible en http://centa.gob.sv
Cisneros-López, ME; Mendoza-Onofre, LE; Mora-Aguilera, G; Córdova-Téllez, L; Livera-Muñoz, M. 2007. Cold tolerant sorghum hybrids and parental lines. I: Seed quality and its effects on seedling establishment. Agrociencia 41:45-55.

Cisneros-López, ME; Mendoza-Onofre, LE; Gonzalez-Hernandez, VA. 2012. Male parent effects on stigma receptivity and seed set of sorghum A-lines under chilling field temperatures. Plant Breeding 131(1):88-93.

Dicko, MH; Gruppen, H; Traoré, AS; Voragen, AGJ; Berkel, WJH. 2006. Sorghum grain as human food in Africa: relevance of content of starch and amylase activities. Afr. J. Biotechnol. 5:384-395.

Dykes, L; Rooney, LW; Waniska, RD; Rooney, WL. 2005. Phenolic compounds and antioxidant activity of sorghum grains of varying genotypes. Journal of Agricultural and Food Chemistry 53:6813-6818.

FAO (Food and Agriculture Organization of the United Nations). 2011. FAOSTAT. ProdStat database, yearly production (en línea). Consultado noviembre 2011. Disponible en http://www.faostat.fao.org

Ferrari, R. 1986. A comparative analysis of the growth of sweet and forage sorghum crops. Dry matter production, phenology and morphology. Australian Journal of Agricultural Research 37:495-512.

Finch-Savage, W.E. 1995. Influence of seed quality on crop establishment, growth and yield. In Basra, AS. ed. Seed quality: basic mechanisms and agricultural implications. Food Products Press, N.Y. USA. p. 361-384.

Flores, NA; Valdés, LCGS. 2009. Formación de líneas de sorgo tolerantes a sequía para la sostenibilidad de los agroecosistemas norestenses. Sociedad Mexicana de Agricultura sostenible. Agricultura Sostenible. Vol. 6. Tuxtla Gutiérrez, Chiapas. México. 423 p.

García, E. 2004. Modificaciones al sistema de clasificación climática de Köeppen. 5 ed. UNAM. Instituto de Geografía. México, D.F. 90 p.

García, JC; Guiragossian, V. 1985. Producción de semilla genética y básica de sorgo. In Taller Producción de Semilla de Sorgo en América Latina, El Batan, Estado de México. p. 82-97.

Gous, F. 1989. Tannins and phenols in black sorghum. Ph. D. Dissertation. Texas A\&M University. College Station, Texas, USA. p. 313-350.

Helms, TC; Deckard, PA; Gregoire, PA. 1997. Corn, sunflower and soybean emergence influenced by soil temperature and soil water content. Agronomy J. 89:59-63. 
Hoffmann-Thoma, G; Hinkel, K; Nicolay, P; Willenbrink, J. 1996. Sucrose accumulation in sweet sorghum stem internodes in relation to growth. Physiologia Plantarum 97(2):277-284.

House, LR. 1985. A guide to sorghum breeding. International Crops Research Institute for the Semi-Arid Tropics. 2 ed. Patancheru. Andhra Pradesh, India. 206 p.

ICRISAT (International Crops Research Institute for the Semi-Arid Tropics). 2009. Annual report 2009. ICRISAT, Patancheru. Andhra Pradesh, India. 68 p.

ISTA (International Seed Testing Association). 2003. International rules for seed testing. Ed. ISTA. Suiza. 550 p.

Lingle, ES. 1987. Sucrose metabolism in the primary culm of sweet sorghum during development. Crop Science 27:1214-1219.

Muminov, NSH. 1997. Dinamics of the accumulation of sugar in sweet sorghum. Chemistry of natural compounds 33(3):353-354.

Murty, UR. 1999. Hybrid seed production in sorghum. In heterosis and hybrid seed production in agronomic crops. Ed. A. S. Barsa. Haworth Press, Inc. USA. p. 119-148.

Olivares, SE. 1994. Paquete estadístico de diseños experimentales FAUANL. Versión 2.5. Facultad de Agronomía UANL. Marín, Nuevo León, México. 15 p.

Patil, RC; Goud, JV. 1980. Viability of pollen and receptivity of stigma in sorghum. Japan Journal of Agricultural Science 50:522-526.

Pfeiffer, TW; Bitzer, MJ; Toy, J; Pedersen, J. 2010. Heterosis in sweet sorghum and selection of a new sweet sorghum hybrid for use in syrup production in A. Crop Sci. 50:1788-1794.

Poehlman, JM. 2005. Mejoramiento genético de las cosechas. 2 ed. Editorial Limusa. D.F., México. 511 p.

Puttarudrappa, A; Goud, JV. 1970. Efficient method for producing hybrid seeds in male-sterile lines. International Sorghum and Millets Newsletter 13:38-39.

Rao, NGP. 2002. Sorghum in evolution and adaptation of cereal crops. Sci. Pub. Inc. E. New. Hampshire. USA. p. $214-238$
Schertz, KF; Dalton, LG. 1980. Sorghum. In Hybridization of crop plants. American Society of Agronomy. Crop Science Society of America. Madison, USA. p. $577-$ 588.

SIAP-SAGARPA (Servicio de Información Agroalimentaria y Pesquera - Secretaría de Agricultura, Ganadería, Desarrollo Rural, Pesca y Alimentación). 2010. Resumen nacional de la producción agrícola. Cultivo de sorgo para grano bajo condiciones de riego y temporal (en línea). Consultado mayo 2010. Disponible en http:// reportes.siap.gob.mx/Agricola_siap

SIAP (Servicio de Información Agroalimentaria y Pesquera). 2011. Resumen nacional de la producción agrícola. Cultivo de sorgo para grano (en línea). Consultado diciembre 2011. Disponible en http://www.siap.gob.mx

SNICS (Servicio Nacional de Inspección y Certificación de Semillas). 2007. Ley Federal de Producción, Certificación y Comercio de Semillas. Ley publicada en el Diario Oficial de la Federación. 15 de Junio, 2007. México, D.F. 17 p.

Tüsüz, MA. 1997. Heterosis in sorghum. In Book of abstracts. The genetics and exploitation of heterosis in crops. An International Symposium. CIMMYT. México, D.F. p. 326-327.

Yu, J; Tuinstra, MR. 2001. Genetic analysis of seedling growth under cold temperature stress in grain sorghum. Crop Sci. 41:1438-1443.

Valdés, LCGS; Pedroza, FJA; José, ECM; Zavala, GF; Juan, VG. 2004. Variedades e híbridos de sorgo de doble propósito vs. híbridos de grano utilizados en sistemas de sorgo-ganado en Nuevo León. In Memorias del XXI Congreso Nacional de Fitogenética. Tuxtla Gutiérrez, Chiapas. México. 231 p.

Williams, AH; Montes, GN; Pecina, QV. 2006. Sorgo. p. 32-54. In Rodríguez del Bosque, LA. ed. Campo Experimental Río Bravo: 50 años de investigación agropecuaria en el norte de Tamaulipas, Historia, Logros y Retos. Capítulo 3. Libro Técnico No. 1. INIFAP. Campo Experimental Río Bravo, Tamaulipas, México. 325 p. 